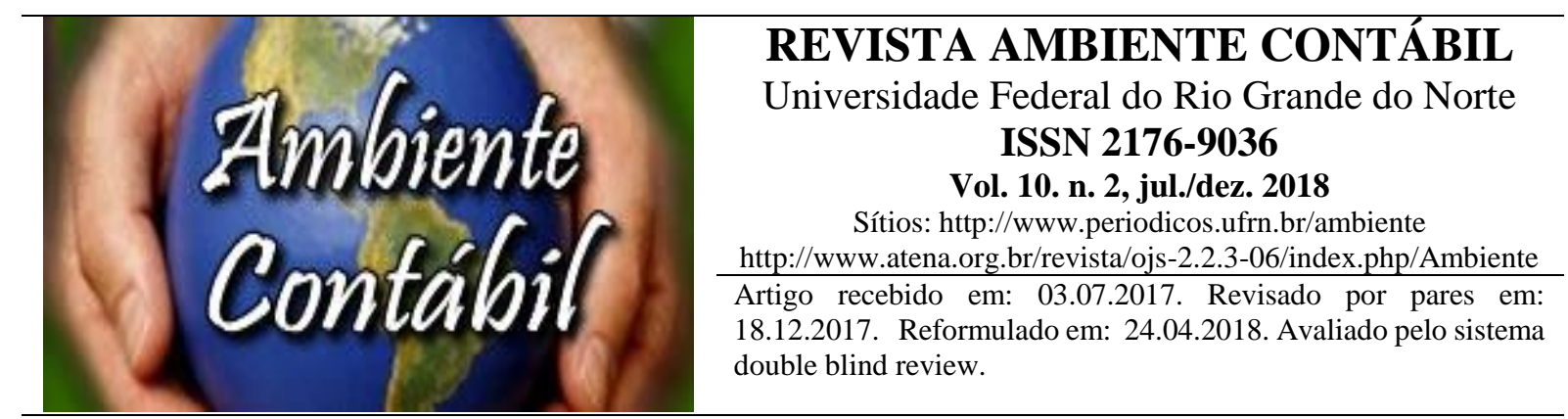

\title{
ANÁLISE DA PERCEPÇÃo DOS DOCENTES QUANTO AOS MÉTODOS BASEADOS EM ATIVIDADES
}

\section{PROFESSOR'S PERCEPTION ANALYSIS REGARDING ACTIVITY-BASED METHODS}

\section{ANÁLISIS DE LA PERCEPCIÓN DE LOS DOCENTES CUANTO A LOS MÉTODOS BASADOS EN ACTIVIDADES}

Autores

\begin{abstract}
Mestrando em Ciências Contábeis - Universidade do Vale do Rio dos Sinos - UNISINOS. Endereço: Av. Unisinos, 950. São Leopoldo/RS - Brasil. Telefone: (51) 35911122.

Identificadores (ID):

Lattes: http://lattes.cnpq.br/5111753697853816

E-mail: marcioprovenzano@gmail.com
\end{abstract}

Mestre em Ciências Contábeis - Universidade do Vale do Rio dos Sinos - UNISINOS. Endereço: Av. Unisinos, 950. São Leopoldo/RS - Brasil. Telefone: (51) 35911122.

Identificadores (ID):

Lattes: http://lattes.cnpq.br/5697669057465290

E-mail: matielcherobini@gmail.com

\begin{abstract}
Rafael Luis Pessin
Mestre em Ciências Contábeis - Universidade do Vale do Rio dos Sinos - UNISINOS. Endereço: Av. Unisinos, 950. São Leopoldo/RS - Brasil. Telefone: (51) 35911122.

Identificadores (ID):

Lattes: http://lattes.cnpq.br/0129088612542075

E-mail: rafaelpessin@outlook.com
\end{abstract}

Débora Gomes Machado

Doutora em Ciências Contábeis e Administração - Professora adjunta da Universidade Federal do Rio Grande - FURG. Endereço: Av. Itália, KM 8, s/ nº Pavilhão 4 - Campus Carreiros Rio Grande - RS - Brasil. Telefone: (53) 3293-5096.

Identificadores (ID): Lattes: http://lattes.cnpq.br/3885093662069571

E-mail: debora_furg@yahoo.com.br 


\begin{abstract}
Marcos Antônio de Souza
Doutor em Controladoria e Contabilidade - Professor da Universidade Municipal de São Caetano do Sul - USCS. Endereço: Rua Santo Antônio, 50. São Caetano do Sul/SP - Brasil.

Telefone: (11) 4239-3200.

Identificadores (ID):

Lattes: http://lattes.cnpq.br/0965183057761983

E-mail: souza.marcosas@gmail.com
\end{abstract}

[Artigo apresentado no XXIII Congresso Brasileiro de Custos, Porto de Galinhas/PE, 2016]

\title{
RESUMO
}

Este estudo buscou analisar a percepção dos docentes e pesquisadores brasileiros quanto aos métodos baseados em atividades. Para tanto, foi realizada uma survey com 34 docentes e pesquisadores especialistas em custos, dos programas de pós-graduação em Ciências Contábeis no Brasil. $\mathrm{Na}$ análise dos resultados foi utilizada a estatística descritiva e empregado o teste de aderência $\left(\chi^{2}\right)$. A consistência interna do instrumento de pesquisa foi calculada por meio do Alpha de Cronbach, o que conferiu consistência ao mesmo. Os resultados demonstram que sob a percepção dos docentes e pesquisadores o Activity Based Cost (ABC) foi capaz de reduzir a subjetividade e arbitrariedade do método por absorção, bem como melhorar as informações para a tomada de decisão. No entanto, o Time-Driven Activity-Based Costing (TDABC) não é considerado de forma unânime uma evolução do método $\mathrm{ABC}$, uma vez que não há uma opinião uníssona se o método foi capaz de reduzir a complexidade e diminuir a necessidade de tempo e recursos relacionados à sua implementação e manutenção. Quanto ao ABM, ele pode ser considerado um sistema de gestão, do qual o ABC constitui sua extensão instrumental.

Palavras-chave: Activity-Based Costing. Time Driven Activity-Based Costing. Activity-Based Management.

ABSTRACT
This study aimed to analyze the perception of Brazilian teachers and researchers regarding activity-based methods. To achieve this goal, we carried out a survey with 34 teachers and researchers of postgraduate programs in Accounting of Brazil. To analyse the results, descriptive statistics and chi-square test $\left(\chi^{2}\right)$ were used. The internal consistency was calculated using Cronbach's Alpha, which gave it consistency. The results demonstrate that $\mathrm{ABC}$ was able to reduce the subjectivity and arbitrariness of the absorption method as well as improve the information for decision making. On the other hand, TDABC is not considered an evolution of the traditional $\mathrm{ABC}$ method, since there is no unanimous opinion as to whether the method was able to achieve some of its objectives, such as reducing complexity and decreasing the need for time and resources related to its implementation and maintenance. With regards to ABM, it can be considered a management system, of which $\mathrm{ABC}$ is your instrumental extension.

Keywords: Activity-Based Costing. Time Driven Activity-Based Costing. Activity-Based Management.

\section{RESUMEN}

Este estudio buscó analizar la percepción de los docentes e investigadores brasileños en cuanto a los métodos basados en actividades. Para ello, se realizó una encuesta con 34 docentes e investigadores especialistas en costos de los programas de postgrado en Ciencias Contables en Brasil. En el análisis de los resultados se utilizó la estadística descriptiva y se empleó la prueba de adherencia $\left(\chi^{2}\right)$. La consistencia interna del instrumento de investigación fue calculada por medio del Alpha de Cronbach lo que confería consistencia al mismo. Los resultados demuestran 
que bajo la percepción de los docentes e investigadores el Activity Based Cost (ABC) fue capaz de reducir la subjetividad y arbitrariedad del método por absorción, así como mejorar las informaciones para la toma de decisión. Sin embargo, el Time-Driven Activity-Based Costing (TDABC) no se considera de forma unánime una evolución del método $\mathrm{ABC}$, ya que no hay una opinión unisona si el método ha sido capaz de reducir la complejidad y disminuir la necesidad de tiempo y tiempo recursos relacionados con su implementación y mantenimiento. En cuanto al ABM, él puede ser considerado un sistema de gestión, del cual el ABC constituye una extensión instrumental.

Palabras clave: Activity-Based Costing. Time Driven Activity-Based Costing. Activity-Based Management.

\section{INTRODUÇÃO}

Nas últimas décadas, os sistemas de contabilidade gerencial foram considerados inadequados à realidade do atual e dinâmico mundo dos negócios. Fatores como a crescente competição global, os rápidos e constantes desenvolvimentos tecnológicos, além da expansão da capacidade de processamento de informações, passaram a exigir dos sistemas contábeis gerenciais a capacidade de fornecer melhores e mais oportunas informações para as atividades de controle de processos, avaliação do custo dos produtos e do desempenho da gestão. (COOPER; KAPLAN, 1992; KAPLAN; ANDERSON, 2004).

À época, consideráveis esforços foram dedicados no desenvolvimento de métodos que pudessem atender as modernas exigências e reduzir as limitações daqueles até então utilizados. No limiar destes avanços, parece não haver dúvidas que um dos maiores impactos sobre a contabilidade gerencial foi promovido pelos métodos baseados em atividades: Activity-Based Costing, Time-Driven Activity-Based Costing e Activity-Based Management. (FRIEDMAN; LYNE, 1997). Estes métodos foram aclamados por prover melhor informação para as decisões estratégicas, planejamento de produtos e gestão de custos (COOPER; KAPLAN, 1988).

Desde os primeiros trabalhos publicados sobre o tema, passando pela popularização do método ABC na década de 80 (MILLER; VOLLMANN, 1985; COOPER; KAPLAN, 1988), temse observado crescente atenção de acadêmicos e gestores sobre as vantagens e benefícios no gerenciamento de custos por meio de atividades. No entanto, uma análise detalhada da literatura desperta questionamentos relevantes sobre estes métodos. Especialmente, sobre tópicos como subjetividade (KAPLAN; ANDERSON, 2007; TSAI et al, 2012; SCHOUTE, 2011), custo/benefício (BYRNE, 2011; MAIGA; NILSSON; JACOBS, 2014; SCHULZE; SEURING; EWERING, 2012; LIN , 2012), complexidade de implantação e manutenção (SANTANA; AFONSO, 2015; SOUZA et al., 2010; EVERAERT et al., 2008; CAMPANALE; CINQUINI; TENUCCI, 2014; GUZMAN et al., 2014; KONT; JANTSON, 2011; STOUTHUYSEN et al., 2010), informação para tomada de decisão (TSAI et al., 2012; SCHOUTE, 2011; BALAKRISHNAN, LABRO, SIVARAMAKRISHMAN, 2012), aplicabilidade (SANTANA; AFONSO, 2015; SOUZA et al., 2010), e até mesmo sobre suas origens (GUNASEKARAN; MCNEIL; SINGH, 2000; KACIUBA; SIEGEL, 2009; ISMAIL, 2010; GOSSELIN, 1997; CAPUSNEANU; MARTINESCU, 2010; PHAN; BAIRD; BLAIRD, 2014).

Estudos como o de Rocha (1994) e Khoury e Ancelevicz (2000) corroboram esta percepção, entretanto, datam de um período já distante e distinto. Em face deste motivo, este estudo indaga se os questionamentos sobre os tópicos controversos dos métodos baseados em atividades já estão consolidados ou ainda se encontram abertos.

Com o intuito de oportunizar novas e revigoradas discussões sobre estes questionamentos, o presente estudo busca analisar a percepção dos docentes e pesquisadores brasileiros quanto aos métodos baseados em atividades. Parte-se da premissa que a academia é um celeiro de conhecimento sobre teorias, conceitos e práticas de gestão, sendo assim, é 
intuitivo imaginar que os docentes e pesquisadores possam contribuir sobremaneira para o debate a respeito desta temática. Salienta-se que não há a intenção de esgotar ou dar como superadas as discussões sobre as controvérsias que envolvem os métodos baseados em atividades, mas oportunizar o avanço dos debates, visto que as discussões anteriores remontam a um passado distante.

Diferentemente dos estudos anteriores (ROCHA, 1994; KHOURY; ANCELEVICZ, 2000), esta pesquisa amplia o enfoque predominantemente teórico sobre as controvérsias dos métodos baseados em atividades, propondo a discutição a partir das respostas de um levantamento com os docentes e pesquisadores especialistas em custos. A partir disto, se espera contribuir com a literatura a partir da reunião de distintas perspectivas sobre o tema e se possível, ousar na direção de uma consolidação de opiniões. Espera-se que este estudo seja concebido mais como um meio de oportunizar novos debates do que um fim em si mesmo.

Além desta seção introdutória, está apresentado na próxima o referencial teórico sobre os temas pesquisados. Na sequência, está delineada a metodologia de pesquisa e, posteriormente, realizada a análise e discussão dos resultados. Encerra-se o estudo com as considerações finais, em que se comentam os principais resultados encontrados.

\section{REFERECIAL TEÓRICO}

\subsection{ACTIVITY-BASED COSTING}

Os fatores relacionados ao aumento da tecnologia dos processos produtivos e do aumento do mix de produtos ocasionou uma diminuição dos custos relativos à mão-de-obra direta e um aumento dos custos indiretos de produção (KAPLAN; ANDERSON, 2007). Como elevados níveis de custos indiretos possuem impacto significativo na lucratividade e competitividade das empresas, o seu gerenciamento deve ser eficiente. (MILLER; VOLLMAN, 1985). Entretanto, os sistemas de custeio baseados em rateio, como o custeio por absorção e o custeio pleno têm apresentado limitações em fornecer informações relevantes para a sua gestão. Balakrishnan, Labro e Sivaramakrishman (2012) acreditam que estes distorcem a realidade dos custos ao impor uma carga elevada de subjetividade e arbitrariedade pelos rateios dos custos indiretos.

Com o intuito de suprir esta limitação, Cooper e Kaplan (1988) desenvolveram o sistema de custeio baseado em atividades (ABC). Diferentemente dos demais, o ABC surge na academia com vistas a preencher lacunas do mercado. Seu principal foco é reduzir os rateios arbitrários dos custos indiretos, presentes nos métodos até então utilizados e que resultam em informações distorcidas que podem levar a decisões equivocadas. A estrutura do modelo $\mathrm{ABC}$ gira em torno do conceito de atividade, entendendo-se ela como a verdadeira causa do consumo de recursos em uma organização. Sob esse enfoque, o ABC busca fornecer informações de custos mais precisas para a tomada de decisão ao rastrear as atividades que o geraram (KAPLAN; ANDERSON, 2007).

Segundo Everaert et al. (2008), o processo de alocação de custos dá-se em duas fases. Na primeira fase, as atividades consomem os recursos. Estes recursos são alocados por meio de Cost Drivers (direcionadores de custos) de recursos, os quais atribuem os custos dos recursos consumidos a cada atividade. Já na segunda fase, as atividades são consumidas pelos objetos de custeio, a partir dos Cost Drivers de atividades. Os objetos de custeio podem ser os próprios produtos fabricados, bem como os serviços ou clientes (EVERAERT et al., 2008).

Durante o processo de alocação, são imputados aos produtos e clientes apenas os custos das atividades realmente consumidas, sendo que a capacidade não utilizada é alocada ao custo de capacidade ociosa (COOPER; KAPLAN, 1992). Este método difere do procedimento adotado pelos modelos tradicionais, os quais imputam os custos totais diretamente aos produtos e clientes, distorcendo as informações. Neste sentido, ao contrário do que ocorre no Custeio por 
Absorção, o ABC não leva em conta o volume de produção (SHANK; GOVINDARAJAN, 1997).

A partir da alocação dos custos baseado nas atividades, o ABC possibilita aos tomadores de decisão uma informação de maior acurácia para determinação do mix de produtos em relação aos métodos tradicionais (TSAI et al., 2012; SCHOUTE, 2011), bem como proporciona uma análise mais adequada da lucratividade de clientes (KIM; KIM, 2011; RATNATUNGA; MICHAEL; BALACHANDRAN, 2012). Não obstante, o ABC permite o custeio da empresa como um todo, mesmo em setores administrativos, sem ligação com o processo produtivo (COOPER; KAPLAN, 1992).

No entanto, apesar dos seus benefícios, o método recebe críticas pela demasiada complexidade de implantação e manutenção, ocasionada pelo excesso de informação, mudanças constantes pela dinamicidade das operações, inclusão e exclusão de produtos, além da necessidade de recursos de tempo e dinheiro. (EVERAERT et al., 2008). Como forma de mitigar estas adversidades, Kaplan e Anderson (2004) desenvolveram o Time-Driven ActivityBased Costing (TDABC), marcando uma importande alteração no sistema $\mathrm{ABC}$ ao buscar simplificar e acelerar o processo de implantação e manutenção.

\subsection{TIME-DRIVEN ACTIVITY-BASED COSTING}

O Time-Driven Activity-Based Costing é um método de custeio que utiliza apenas o tempo como direcionador de custos. Em sua essência, o TDABC é formado por um conjunto de equações de tempo, estimadas com base em experiências históricas que demonstram como diversas operações e demandas consomem capacidade de recursos. A atualização destas equações é realizada a partir dos ganhos de produtividade derivados de processos de melhoria (KAPLAN; NORTON, 2008). Conforme Kaplan e Anderson (2004), são necessários apenas dois parâmetros para sua implementação: o custo necessário para fornecer a capacidade das atividades e o tempo necessário para a realização de uma atividade.

O desenvolvimento do TDABC trouxe como benefício a possibilidade de ajustes aos diferentes níveis de complexidade das atividades, podendo ser capturada uma ampla quantidade de tarefas (sub-atividades) a serem inseridas nas equações de tempo (EVERAERT et al., 2008). $\mathrm{O}$ método também diminui a necessidade de entrevistas, bem como permite a visualização da capacidade de recursos humanos e tecnológicos ociosos. (DALCI; TANIS; KOSAN, 2010; BOINA; SOUZA; AVELAR, 2015).

As premissas relacionadas ao TDABC têm sido estudadas por diferentes autores. (GUZMAN et al., 2014; KONT; JANTSON, 2011; STOUTHUYSEN et al.; 2010). Estes estudos corroboram alguns dos benefícios apontados por Kaplan e Anderson (2004), pois demonstram que esta nova abordagem é mais rápida, fácil e menos onerosa do que o método $\mathrm{ABC}$ tradicional. Entretanto, algumas destas vantagens não são consensos em outras pesquisas sobre o tema, ao apresentarem dificuldades para a formulação das equações de tempo, grande volume de dados para a sua validação e elevado desvio padrão no tempo de execução das atividades. (SOUZA et al., 2010; SANTANA; AFONSO, 2015).

De forma geral, o desenvolvimento do TDABC trouxe melhorias consideráveis ao método ABC. Entretanto, conforme Shank e Govindarajan (1997) ambos os métodos atribuem custos aos produtos sem qualquer preocupação quanto à legitimidade dos custos em termos estratégicos. Os autores suportam um novo enfoque na redução ou eliminação de todos os custos que não forem valores agregados. Assim, emerge a necessidade de um sistema que reconheça a necessidade de repensar continuamente que atividades agregam valor para o cliente e como desempenhar estas de maneira mais eficiente: o Activity-Based Management. 


\subsection{ACTIVITY-BASED MANAGEMENT}

O Activity-Based Management é um sistema que foca na gestão de atividades no âmbito dos processos de negócios com o objetivo de melhorar continuamente o valor entregue aos clientes e os lucros auferidos no fornecimento deste valor (GHICAJANU, 2008). O conceito de atividade é o fator central do ABM, o modelo utiliza informações de custos baseadas em atividades e medidas de desempenho para influenciar a ação da gestão. Pessoas realizam atividades que consomem recursos, assim, controlar as atividades permite ao gestor controlar os custos na sua fonte (KREN, 2008).

Diferentemente da visão vertical dos métodos de custeio, a organização é entendida como um sistema composto de atividades que se relacionam entre si. Neste contexto, a empresa deve ser vista não apenas por meio das suas funções, mas também por meio de seus processos (GHICAJANU, 2008). Tardivo, Montezemolo e Cordero (2009) corroboram este entendimento ao afirmar que o ABM é baseado no fato de que uma vantagem competitiva é conquistada abandonando o ponto de vista do produto (função), focando no controle dos processos ao longo da cadeia de valor.

A análise de atividades proporcionada pelo ABM consiste em identificar os procedimentos que transformam recursos em produtos e serviços, bem como seus custos e os fatores que os causaram (GOSSELIN, 1997). Envolve a identificação das atividades críticas, das atividades geradoras e não geradoras de valor e o benchmarking (GUNASEKARAN; MCNEIL; SINGH, 2000). Esta análise é importante, pois possibilita uma adequada compreensão das tarefas e dos processos realizados, melhorando a eficiência e eficácia das atividades (PHAN; BAIRD; BLAIRD, 2014).

Ademais, o ABM é associado à melhor gestão dos recursos, por meio da identificação das atividades geradoras de custos (GHICAJANU, 2008; ROZTOCKI, 2010; KREN, 2008); à redução de custos pela melhoria dos processos (BAIRD; HARRISON; REEVE, 2004, KREN, 2008); ao controle do desempenho por meio de medidas financeiras e não financeiras (GUNASEKARAN; MCNEIL; SINGH, 2000; CAPUSNEANU; MARTINESCU, 2010; GHICAJANU, 2008), à melhoria da tomada de decisão, por meio da análise da lucratividade de clientes e linhas de produtos (ROZTOCKI, 2010), além da melhora do desempenho pela integração com outros modelos de gestão (HIXON, 1995; CAPUSNEANU; MARTINESCU, 2010).

No contexto da literatura sobre métodos baseados em atividades, alguns estudos definem o ABM como uma ferramenta gerencial originada do Activity-based Costing, na medida em que se beneficia de informações proporcionadas pelo ABC para gerir as atividades (GUNASEKARAN; MCNEIL; SINGH, 2000; KACIUBA; SIEGEL, 2009; ISMAIL, 2010). Entretanto, outros autores entendem que o método $A B C$ representa uma extensão instrumental do modelo ABM (GOSSELIN, 1997; CAPUSNEANU; MARTINESCU, 2010; PHAN; BAIRD; BLAIRD, 2014). Percebe-se assim, que as opiniões são divididas.

\subsection{QUESTIONAMENTOS SOBRE OS MÉTODOS BASEADOS EM ATIVIDADES}

Apesar das vantagens e benefícios apontados até o momento sobre os métodos baseados em atividades, uma análise mais detalhada da literatura desperta a atenção para questionamentos relevantes sobre os mesmos. Buscou-se, a partir da revisão de artigos sobre a temática, relacionar tópicos nos quais foram evidenciados resultados contrastantes entre estudos, inclusive entre teoria e prática. O Quadro 1 relaciona os questionamentos com seus respectivos autores. 


\section{Quadro 1 - Questionamentos sobre os métodos baseados em atividades}

\begin{tabular}{|c|c|c|}
\hline Tópico & Questionamentos & Autores \\
\hline Subjetividade & $\begin{array}{l}\text { Um dos principais objetivos dos métodos baseados em atividades } \\
\text { é reduzir os rateios arbitrários que se fazia uso no método por } \\
\text { absorção, o que lhe conferia demasiada subjetividade. Entretanto, } \\
\text { ainda há questionamentos quanto à subjetividade dos } \\
\text { direcionadores de custo e atividades utilizados por estes métodos. }\end{array}$ & $\begin{array}{l}\text { Kaplan e Anderson (2007); Tsai } \\
\text { et al. (2012); Schoute (2011). }\end{array}$ \\
\hline $\begin{array}{l}\text { Custo x } \\
\text { Benefício }\end{array}$ & $\begin{array}{l}\text { Apesar de haver certa unanimidade quanto à necessidade de } \\
\text { recursos (tempo e dinheiro) para a implantação do ABC e } \\
\text { TDABC, há questionamentos quanto à relação entre o montante } \\
\text { de custos incorridos e os benefícios futuros. }\end{array}$ & $\begin{array}{l}\text { Byrne (2011); Maiga, Nilsson e } \\
\text { Jacobs (2014); Schulze, Seuring, } \\
\text { Ewering (2012); Lin (2012). }\end{array}$ \\
\hline Complexidade & $\begin{array}{l}\text { A complexidade na implantação e manutenção dos métodos } \\
\text { ABC e TDABC é um dos tópicos mais controversos nas } \\
\text { pesquisas. A extensão das entrevistas, volume de dados, } \\
\text { necessidade de recursos e adesão da equipe ainda não são } \\
\text { totalmente esclarecidos nos estudos. }\end{array}$ & $\begin{array}{l}\text { Santana e Afonso (2015); Souza } \\
\text { et al. (2010); Everaert et al. } \\
\text { (2008); Campanale, Cinquini e } \\
\text { Tenucci (2014); Guzman et al. } \\
\text { (2014); Kont e Jantson (2011); } \\
\text { Stouthuysen et al. (2010). }\end{array}$ \\
\hline $\begin{array}{l}\text { Tomada de } \\
\text { Decisão }\end{array}$ & $\begin{array}{l}\text { Ao incluir os direcionadores de custos o método ABC busca } \\
\text { melhorar a informação para a tomada de decisão se comparado } \\
\text { aos métodos tradicionais, havendo questionamentos se o mesmo } \\
\text { foi capaz de proporcionar isto. Também é recorrente a questão se } \\
\text { a utilização do tempo como direcionador pelo TDABC pode } \\
\text { suprir esta demanda de informações. }\end{array}$ & $\begin{array}{l}\text { Tsai et al. (2012); Schoute (2011); } \\
\text { Balakrishnan, Labro e } \\
\text { Sivaramakrishman (2012). }\end{array}$ \\
\hline Aplicação & $\begin{array}{l}\text { Os estudos sobre a adoção na prática do } \mathrm{ABC} \text { e TDABC ainda } \\
\text { geram dúvidas sobre a adequação dos métodos às diferentes } \\
\text { organizações e ramos de atividades. Apesar de alguns indicativos, } \\
\text { falta uma compreensão mais proeminente sobre a temática. }\end{array}$ & $\begin{array}{l}\text { Santana e Afonso (2015); Souza } \\
\text { et al. (2010). }\end{array}$ \\
\hline Origem & $\begin{array}{l}\text { A origem do } \mathrm{ABC} \text { e TDABC parece estabelecida na literatura, } \\
\text { entretanto, o mesmo não pode ser dito do } \mathrm{ABM} \text {. Sua concepção } \\
\text { é entendida tanto como uma ferramenta originada do } \mathrm{ABC} \text {, } \\
\text { quanto um modelo no qual o } \mathrm{ABC} \text { é apenas uma extensão } \\
\text { instrumental. }\end{array}$ & $\begin{array}{l}\text { Gunasekaran, Mcneil e Singh } \\
\text { (2000); Kaciuba e Siegel (2009); } \\
\text { Ismail (2010); Gosselin (1997); } \\
\text { Capusneanu e Martinescu (2010); } \\
\text { Phan, Baird e Blaird (2014). }\end{array}$ \\
\hline
\end{tabular}

Fonte: Elaborado pelos autores.

Conforme observado no Quadro 1, especificamente, observam-se questionamentos quanto à subjetividade, informação para tomada de decisão, complexidade de implantação e manutenção, custo/benefício, aplicabilidade e até mesmo a origem dos métodos.

\section{PROCEDIMENTOS METODOLÓGICOS}

Para atingir o objetivo proposto, foi realizada uma pesquisa descritiva com abordagem quantitativa. Os dados foram coletados mediante aplicação de um questionário com 10 afirmações, enviado por e-mail aos docentes e pesquisadores especialistas em custos dos programas de pós-graduação em ciências contábeis brasileiros. Por meio de uma escala tipo Likert de sete pontos, os respondentes foram solicitados a mensurar o seu grau de concordância ou discordância em relação às afirmativas. Os enunciados foram elaborados a partir dos questionamentos e autores base de cada enfoque relacionados no Quadro 1.

A estruturação da escala foi idealizada tendo em vista o elevado grau de conhecimento dos docentes em relação ao assunto e com o intuito de incentivar o posicionamento dos mesmos. Diferentes autores (MATELL; JACOBY, 1972; COELHO; ESTEVES, 2007) associam as escalas de sete pontos a um menor nível de uso da resposta intermediária, à maior habilidade dos respondentes e a um maior poder explanatório. Ressalta-se que o instrumento de pesquisa foi previamente testado com quatro docentes (não participantes da amostra final) e posteriormente 
ajustado para ser enviado. Os ajustes se concentraram, essencialmente, na redação e clareza textual das afirmações.

O universo deste estudo foi composto pelos docentes dos programas de pós-graduação (PPGs) - mestrado profissional, mestrado acadêmico e doutorado - em Ciências Contábeis do Brasil, recomendados pela Coordenação de Aperfeiçoamento de Pessoal de Nível Superior (CAPES). Conforme a CAPES, no ano de 2016 existiam no Brasil 26 programas de pósgraduação. Após acesso aos endereços eletrônicos de cada programa, foram evidenciados 342 professores vinculados aos respectivos PPGs. Não fizeram parte do levantamento os docentes da Universidade Federal do Rio Grande do Sul, em função da mesma não divulgar os professores titulares do respectivo programa.

Deste montante, foram selecionados mediante análise de conteúdo do Currículo Lattes, os docentes e pesquisadores especialistas em custos de acordo com os seguintes critérios de inclusão: a) disciplinas ministradas relacionadas à custos; b) linha de pesquisa relacionada à custos; c) área de atuação relacionada à custos; e; d) trabalhos publicados relacionados à custos. Para as disciplinas ministradas e trabalhos publicados, foi analisado o período de 2012 a 2016, sendo a existência de uma única ocorrência nestes critérios considerada suficiente para a sua inclusão. Ressalta-se que todos os critérios foram analisados de forma independente, assim, o atendimento a qualquer um dos mesmos foi considerado suficiente para a inclusão do docente ou pesquisador. Em face do exposto, foram identificados 141 professores que obedeciam aos requisitos pré-estabelecidos.

O primeiro e-mail foi enviado no dia 20 de Junho de 2016, resultando em 22 respostas completas. No dia 27 de Junho de 2016, um segundo envio foi efetuado, direcionado aos não respondentes, resultando em 12 respostas completas. A amostra foi definida, assim, pelo somatório dos retornos, totalizando 34 docentes, conforme Tabela 1. Esta representa 24,1\% de todos os potenciais respondentes e pode ser considerada adequada de acordo com os padrões recomendados para este tipo de instrumento. (NULTY, 2008). Como forma de validar a amostra, foi utilizado o procedimento proposto por Diehl, Gonçalo e Martins (2002). As respostas para a questão 2 (escolhida aleatoriamente) obtidas nos dois envios foram comparadas. Os valores das médias, desvios-padrão, moda e mediana não apresentaram diferenças significativas, fato que valida a amostra no sentido da qualidade da informação.

Tabela 1 - Status de resposta do instrumento

\begin{tabular}{|c|c|c|c|c|c|}
\hline \multirow{2}{*}{ Região } & \multicolumn{2}{|c|}{ Enviados } & \multicolumn{2}{|c|}{ Respondidos } & \multirow{2}{*}{$\begin{array}{c}\% \\
\text { Amostra }\end{array}$} \\
\hline & IES & Docentes & IES & Docentes & \\
\hline Nordeste & 4 & 22 & 3 & 3 & $9 \%$ \\
\hline Centro-Oeste & 2 & 17 & 1 & 2 & $6 \%$ \\
\hline Sudeste & 12 & 63 & 8 & 13 & $38 \%$ \\
\hline Sul & 7 & 39 & 7 & 16 & $47 \%$ \\
\hline Total & 25 & 141 & 19 & 34 & $100 \%$ \\
\hline
\end{tabular}

Fonte: Elaborada pelos autores.

Do total de respondentes que compõe a base amostral de pesquisa, foram vinculados 19 programas de pós-graduação. Estes PPGs são relacionados no Quadro 2. 


\section{Quadro 2 - Programas de pós-graduação integrantes da amostra do estudo}

\begin{tabular}{|l|l|c|l|}
\hline \multicolumn{1}{|c|}{ PPGs } & \multicolumn{1}{c|}{ IES } & UF & \multicolumn{1}{c|}{ Tipo } \\
\hline Contabilidade & UFBA & BA & Mestrado Acadêmico \\
\hline Ciências Contábeis & UNB & DF & Doutorado e Mestrado Acadêmico \\
\hline Controladoria e Contabilidade & UFMG & MG & Mestrado Acadêmico \\
\hline Ciências Contábeis & UFU & MG & Mestrado e Doutorado Acadêmico \\
\hline Ciências Contábeis & UFPB/J.P. & PB & Mestrado e Doutorado Acadêmico \\
\hline Ciências Contábeis & UEM & PR & Mestrado Acadêmico \\
\hline Contabilidade & UNIOESTE & PR & Mestrado Acadêmico \\
\hline Contabilidade & UFPR & PR & Doutorado e Mestrado Acadêmico \\
\hline Ciências Contábeis & UFRJ & RJ & Mestrado e Doutorado Acadêmico \\
\hline Ciências Contábeis & UFRN & RN & Mestrado Acadêmico \\
\hline Ciências Contábeis & UNISINOS & RS & Doutorado e Mestrado Acadêmico \\
\hline Ciências Contábeis & FURB & SC & Doutorado e Mestrado Acadêmico \\
\hline Contabilidade & UFSC & SC & Doutorado e Mestrado Acadêmico \\
\hline Ciências Contábeis e Administração & UNOCHAPECÓ & SC & Mestrado Acadêmico \\
\hline Ciências Contábeis e Atuariais & PUC/SP & SP & Mestrado Acadêmico \\
\hline Ciências Contábeis & UniFECAP & SP & Mestrado Acadêmico \\
\hline Controladoria Empresarial & UPM & SP & Mestrado Profissional \\
\hline Controladoria e Contabilidade & USP & SP & Doutorado e Mestrado Acadêmico \\
\hline Controladoria e Contabilidade & USP/RP & SP & Doutorado e Mestrado Acadêmico \\
\hline
\end{tabular}

Fonte: Elaborado pelos autores com base nos endereços eletrônicos dos programas de pós-graduação.

Para apresentar os resultados, foi utilizada a estatística descritiva através das distribuições de frequências absolutas, medidas de tendência central (média, moda e mediana) e desvios-padrão. Adicionalmente, como forma de reforçar os resultados descritivos, foi empregado o teste de aderência $\left(\chi^{2}\right)$. Neste, os respondentes foram agrupados em três categorias, conforme o grau de concordância para cada uma das questões: não concordo (escores de 1 a 3), indiferente (escore 4) e concordo (escores de 5 a 7). A partir deste método, pretendeu-se verificar se as frequências de resposta para as categorias eram significativamente diferentes das frequências esperadas. (HAIR et al., 2005). Ademais, a consistência interna do instrumento de pesquisa foi calculada por meio do Alpha de Cronbach (CRONBACH, 1951), sendo este estimado em 0,70; o que confere consistência ao mesmo.

\section{ANÁLISES E DISCUSSÕES}

Esta seção é segmentada de acordo com os tópicos vinculados aos questionamentos sobre os métodos baseados em atividades elencados no Quadro 1. Para consecução das análises a Tabela 2 apresenta a estatística descritiva, além do teste de aderência para cada tópico. 
Tabela 2 - Estatística sobre os questionamentos dos métodos baseados em atividades

\begin{tabular}{|c|c|c|c|c|c|c|c|c|}
\hline Questionamentos & Disc. & Indif. & Conc. & Média & D.P. & Moda & Mediana & $x^{2}$ \\
\hline 1-Subjetividade $\mathrm{ABC}$ & 8 & 4 & 22 & 4,62 & 1,78 & 6 & 5 & $6,902 *$ \\
\hline 2-Subjetividade TDABC & 25 & 2 & 7 & 2,71 & 1,59 & 2 & 2 & $13,078 * *$ \\
\hline 3-Custo $x$ benefícios ABC & 14 & 12 & 8 & 3,56 & 1,19 & 4 & 4 & $13,490 * *$ \\
\hline 4-Complexidade $\mathrm{ABC}$ & 2 & 2 & 30 & 5,97 & 1,09 & 6 & 6 & $28,863 * *$ \\
\hline 5-Complexidade TDABC & 12 & 5 & 17 & 4,38 & 1,63 & 5 & 4,5 & 0,863 \\
\hline 6-Tomada de decisão $A B C$ & 6 & 1 & 27 & 5,26 & 1,64 & 6 & 6 & $18,706^{* * *}$ \\
\hline 7-Tomada de decisão TDABC & 13 & 4 & 17 & 4,03 & 1,57 & 5 & 4,5 & 0,725 \\
\hline 8-Aplicação TDABC & 25 & 1 & 8 & 2,71 & 1,78 & 2 & 2 & $13,490 * *$ \\
\hline 9-Origem ABM & 12 & 6 & 16 & 4,21 & 1,81 & 6 & 4 & 0,863 \\
\hline 10- $\mathrm{ABC}$ versus $\mathrm{TDABC}$ & 19 & 5 & 10 & 3,53 & 1,88 & 2 & 3 & 2,784 \\
\hline
\end{tabular}

Nota: Disc.: Discordo; Indif.: Indiferente; Conc.: Concordo; $(* *)$ significativo $p \leq 0,01 ;(* *)$ significativo $p \leq 0,05$.

Fonte: Elaborado pelos autores.

\section{- SUBJETIVIDADE}

Recuperar a relevância das informações de custos perdida pelo custeio por absorção frente ao crescimento dos custos indiretos, segundo Kaplan e Anderson (2007), foi um dos principais motivos para o surgimento do método $\mathrm{ABC}$. Nesse sentido, questionou-se se o método de fato contribuiu para resolver o problema relacionado à subjetividade dos rateios arbitrários utilizados pelo custeio por absorção. As respostas com relação à subjetividade do $\mathrm{ABC}$ (Item 1 da Tabela 2) tiveram média de 4,62 e moda de 6,00, uma leve tendência à concordância. Para atestar esta percepção, foi utilizado o teste de aderência $\left(x^{2}\right)$ com o intuito de verificar se há diferenças significativas entre as categorias de concordância (discordo, indiferente e concordo). Como observado no teste empregado, os resultados sugerem haver, de fato, concordância dos respondentes $\left(x^{2}=7,309 ; \mathrm{p} \leq 0,05\right)$. Estes resultados corroboram a visão de Kaplan e Anderson (2007); os docentes participantes desta pesquisa entendem que o $\mathrm{ABC}$ foi capaz de reduzir tal subjetividade, conferindo maior relevância a suas informações.

Já o método TDABC aparenta não possibilitar uma melhora na subjetividade das informações. Questionados se a utilização do tempo como único direcionador de custos explica perfeitamente as atividades da empresa, gerando informações precisas sobre os custos, os docentes demonstraram discordância. $\mathrm{O}$ item 2 da Tabela 2 apresenta média para as respostas de 2,71 e mediana e moda de 2,00, logo, opinião contrária à afirmação. respectivamente. O teste de aderência corrobora esta percepção ao mostrar significância $\left(x^{2}=13,078 ; \mathrm{p} \leq 0,01\right)$. Estes resultados denotam uma baixa concordância por parte dos docentes pesquisados sobre a capacidade deste método em gerar informações precisas e objetivas sobre os custos. O estudo de Varila, Seppänen e Suomala (2007) reforçam estes achados. A utilização das equações de tempo, na ocasião, não foi capaz de explicar de maneira satisfatória o tempo necessário para a execução das atividades.

\section{- CUSTO X BENEFÍ́CIO}

Outro ponto de destaque está na discussão sobre a relação entre custos versus benefícios dos métodos baseados em atividades, especialmente o ABC. Conforme o Quadro 1, há questionamentos quanto à relação entre o montante de custos incorridos e os benefícios futuros. De acordo com o item 3 da Tabela 2, a percepção dos docentes no tocante aos benefícios gerados pelo $\mathrm{ABC}$ frente aos recursos de tempo e dinheiro consumidos para sua implantação e manutenção não possui concordância. As respostas apresentaram média de 3,56, com moda e mediana de 4,00. $\mathrm{O}$ 
teste de aderência corrobora ao se mostrar estatisticamente significante $\left(x^{2}=13,490 ; p \leq 0,01\right)$, visto $\mathrm{o}$ alto número de respostas intermediárias.

Essa tendência central de respostas não infere concordância nem discordância com o retorno auferido pela implantação do método. Esse resultado mostra-se condizente com a literatura, onde existem diferentes pontos de vista. Autores como Byrne (2011) e Maiga, Nilsson e Jacobs (2014) apontam que os recursos investidos, principalmente nas fases iniciais de implantação são altos, além de o processo ser demasiadamente lento. Já para outros, como Schulze, Seuring, Ewering (2012) e Lin (2012), os benefícios tendem a superar os investimentos.

\section{- COMPLEXIDADE}

Conforme o Quadro 1, a complexidade na implantação e manutenção dos métodos $\mathrm{ABC}$ e TDABC é um dos tópicos mais controversos nas pesquisas. No $\mathrm{ABC}$, autores como Byrne (2011) e Schulze, Seuring e Ewering (2012) apontam que o método apresenta grande complexidade para sua implementação e manutenção. Bokor e Somogyi (2015) corroboram este entendimento e destacam que o método apresenta uma maior necessidade de entrada de dados quando comparado aos métodos de custeio anteriores. Para os docentes e pesquisadores brasileiros há a mesma percepção sobre a demasiada complexidade do método $\mathrm{ABC}$. Conforme a Tabela 2, as respostas para a complexidade do $\mathrm{ABC}$ (Item 4) apresentou média de 5,97 e mediana e moda de 6,00. Observa-se também, que as respostas para este questionamento possuem o menor desvio padrão da pesquisa. Esta percepção é reforçada pelos resultados significativos do teste de aderência $\left(x^{2}=28,863 ; \mathrm{p} \leq 0,01\right)$. Ou seja, a frequência de respostas para a categoria de indivíduos que concordam com esta afirmação é significativamente diferente das demais categorias.

Como forma de mitigar a complexidade reportada pelo ABC, Kaplan e Anderson (2004) desenvolveram o método TDABC. Para os respondentes desta pesquisa, a Tabela 2 (Item 5) evidencia uma leve tendência em considerar que os processos de implantação e manutenção do método TDABC, propostos pelos autores, realmente exigem menos recursos de tempo e dinheiro que o método ABC. Os resultados demonstram média de 4,38, mediana de 4,50 e moda em 5,00. No entanto, destaca-se que a proporção de respostas entre as diferentes categorias de concordância não apresentou significância; este fato contraria os resultados descritivos e não permite uma inferência sobre a opinião dos docentes quanto ao TDABC e a sua intenção de reduzir a complexidade do método $\mathrm{ABC}$.

Estes achados refletem os resultados encontrados na literatura, os quais demonstram interpretações divergentes quanto aos processos de implantação e manutenção do método TDABC. Ou seja, não houve clareza neste ponto. Enquanto estudos como os de Campanale, Cinquini e Tenucci (2014), Guzman et al. (2014), Kont e Jantson (2011) e Stouthuysen et al. (2010), inferem que o método TDABC é mais facil e rápido de implementar que o $\mathrm{ABC}$; outros, como os de Santana e Afonso (2015) e Everaert et al. (2008) apresentam como limitação justamente a necessidade de um grande volume de dados para sua validação, além da realização de extensas entrevistas. Isto demandaria uma grande quantidade de recursos, principalmente de tempo e pessoas para a sua implantação, anulando o objetivo do seu desenvolvimento.

\section{- TOMADA DE DECISÃO}

No âmbito das informações para a tomada de decisão, os sistemas tradicionais de custeio têm apresentado limitações em fornecer informações relevantes para a sua gestão. Balakrishnan, Labro e Sivaramakrishman (2012) acreditam que os mesmos distorcem a realidade dos custos, na medida em que impõem uma carga elevada de subjetividade e arbitrariedade pelos rateios dos custos indiretos. Na expectativa de suprir este problema, o ABC 
foi desenvolvido. Tal objetivo parece ter se concretizado na percepção dos docentes e pesquisadores. Segundo estes, em comparação ao método de custeio por absorção, o método ABC proporciona melhor e mais precisa informação para a gestão dos custos.

As respostas relacionadas na Tabela 2 (Item 6) apresentam média de 5,26, mediana e moda de 6,00 , respectivamente. Soma-se a isto o fato do teste de aderência ter se mostrado estatisticamente significativo $\left(x^{2}=18,706 ; \mathrm{p} \leq 0,01\right)$, com maior proporção de respostas positivas. Estes resultados vão ao encontro de estudos que evidenciam a superioridade informacional do método ABC (TSAI et al., 2012; LIN, 2012), possibilitando aos tomadores de decisão uma informação de maior acurácia para determinação do mix de produtos (TSAI et al., 2012; SCHOUTE, 2011), bem como uma análise mais adequada da lucratividade de clientes (KIM; KIM, 2011; RATNATUNGA; MICHAEL; BALACHANDRAN, 2012).

Por outro lado, as informações de tempo proporcionadas pelo TDABC, apesar de buscarem simplificar e agilizar a implantação e manutenção do método $\mathrm{ABC}$, parecem não ter a mesma acurácia, limitando a tomada de decisão. Como visto no item 2 anteriormente, a utilização do tempo como único direcionador de custos no TDABC não explica perfeitamente as atividades da empresa, não gerando informações precisas sobre os custos. Estes achados reforçam os resultados encontrados por Varila, Seppänen e Suomala (2007). Os autores apontam que a utilização das equações de tempo não foi capaz de explicar de maneira satisfatória o tempo necessário para a execução das atividades, impossibilitando decisões eficazes.

Soma-se a isto o fato de não haver concordância de que o método TDABC proporciona melhores informações a respeito da capacidade ociosa. As respostas para o item 7, embora tenham apresentado média 4,03, mediana 4,50 e moda 5,00, não mostraram significância no teste de aderência. Estes resultados contrariam os estudos de Dalci, Tanis e Kosan (2010) e Boina, Souza e Avelar (2015), os quais afirmam que o maior benefício apresentado pelo TDABC é justamente a identificação da capacidade ociosa.

As evidentes limitações relacionadas ao TDABC podem justificar a percepção dos docentes quanto ao item 10. Questionados se a simplificação proposta pelo método, a partir da utilização do tempo como único direcionador de custos, é um retrocesso ao $\mathrm{ABC}$, os respondentes não chegaram a uma conclusão definitiva. Embora os resultados para este item tenham apresentado média de 3,53, moda de 2,00 e desvio-padrão de 1,88. (1,88), a falta de significância no teste de aderência demostra ausência de uma opinião consolidada. Em suma, não há subsídios para afirmar que o método é uma evolução natural do $\mathrm{ABC}$, sendo a utilização do tempo como único direcionador uma inovação conceitual positiva.

\section{- APLICAÇÃO}

A aplicação dos métodos baseados em atividades tem recebido grande atenção desde os primeiros estudos publicados sobre o tema (KAPLAN; ANDERSON, 2007). Apesar das considerações sobre a complexidade de implantação, comentadas anteriormente, os métodos tendem a ser percebidos com maior intensidade em empresas com maior proporção de custos indiretos e com maior complexidade na linha de produtos (SHANK; GOVINDARAJAN, 1997). Estes determinantes, justamente, foram apontados por Cooper e Kaplan (1988) como fatores motivadores para o desenvolvimento do método $\mathrm{ABC}$.

Especificamente, estudos sobre a adoção dos métodos baseados em atividades evidenciam que a adoção do $\mathrm{ABC}$ é associada a organizações mecanicistas e com maior nível de diferenciação vertical. Por sua vez, as práticas de análises de atividades como forma de gestão, mais próximas à concepção do ABM, são associadas a empresas orgânicas. (GOSSELIN, 1997; BAIRD; HARRISON; REEVE, 2004; PHAN; BAIRD; BLAIR, 2014). Entretanto, pouca atenção tem sido dispendida ao estudo sobre a implementação do método TDABC, ocasionando dúvidas sobre a sua aplicação. 
Para os docentes pesquisados, o método TDABC não é adequado a todos os tipos de organizações e ramos de atividade. Os resultados apontados pela Tabela 2 (Item 8) evidenciam média de 2,71 e moda de 2,00. Atesta-se esta opinião negativa pelos resultados estatisticamente significantes do teste de aderência $\left(x^{2}=13,490 ; p \leq 0,01\right)$. Este fato pode estar relacionado ao entendimento de que a utilização do tempo como único direcionador de custos não explica perfeitamente as atividades de todas as empresas, podendo gerar informações imprecisas sobre os custos (Item 2). Da mesma forma, pode estar relacionado à dificuldade para a formulação das equações de tempo e ao elevado desvio padrão no tempo de execução das atividades. (VARILA; SEPPÄNEN; SUOMALA, 2007; SOUZA et al., 2010; SANTANA; AFONSO, 2015).

\section{- ORIGEM}

A consideração da origem dos métodos baseados em atividades como questionamento é um dos tópicos menos controversos analisados neste estudo. Parece consolidado que as discussões sobre atividades remontam ao final da década de 70 e início de 80 , tendo como exemplo o trabalho de Miller e Vollmann (1985) sobre transações. O método ABC, no entanto, veio a ser notavelmente reconhecido apenas após o artigo Measure Costs Right: Make the Right Decisions de Cooper e Kaplan (1988). Da mesma forma, parece estabelecido que o TDABC foi apresentado por Kaplan e Anderson (2004), como resposta às dificuldades enfrentadas pelas organizações na implementação do ABC.

Entretanto, consideráveis questionamentos podem ser feitos sobre a gestão baseada em atividades. Para os docentes pesquisados, item 9, há uma maior tendência de considerar o método ABC como uma extensão instrumental do sistema de gestão $\mathrm{ABM}$, média de 4,21, porém com moda de 6,00. Estes resultados contrariam estudos que definem o sistema como uma ferramenta gerencial originada do $\mathrm{ABC}$, na medida em que se beneficia de informações proporcionadas pelo mesmo para gerir as atividades. (GUNASEKARAN; MCNEIL; SINGH, 2000; KACIUBA; SIEGEL, 2009; ISMAIL, 2010). Cabe salientar, no entanto, que o teste de aderência não mostrou significância entre as proporções de respostas.

Acredita-se que a percepção dos docentes se aproxima dos trabalhos de Gosselin (1997) e Capusneanu e Martinescu (2010). Para Gosselin (1997), o ABM é entendido a partir de três níveis de atividade: Análise de atividades (AA); análise dos custos das atividades (ACA) e custeio baseado em atividades (ABC). Nos níveis iniciais (AA e ACA) o propósito é melhorar os processos e a eficiência em custo; subsequente, o nível avançado (ABC) proporciona o rastreamento dos custos aos produtos e serviços. Sob esta perspectiva, o ABC é entendido como um instrumento de custeio.

\section{CONSIDERAÇÕES FINAIS}

Este estudo teve como objetivo analisar a percepção dos docentes e pesquisadores brasileiros quanto aos métodos baseados em atividades. Para tanto, foi realizado um levantamento com 34 docentes e pesquisadores especialistas em custos de 19 programas de pós-graduação em Ciências Contábeis. Partiu-se da premissa de que a academia é um celeiro de conhecimento sobre teorias, conceitos e práticas de gestão, sendo assim, é intuitivo imaginar que os docentes e pesquisadores pudessem contribuir sobremaneira para o debate a respeito da temática. Os resultados foram analisados mediante estatística descritiva e teste de aderência $\left(x^{2}\right)$.

A partir dos resultados encontrados no levantamento, foi possível observar que o método de custeio $\mathrm{ABC}$, quando comparado ao método de custeio por absorção, foi capaz de atingir seus objetivos segundo os docentes pesquisados, possibilitando a redução das subjetividades impostas pelo método anterior bem como melhorando as informações para a tomada de decisão. Entretanto, foi possível perceber também a demasiada complexidade do 
$\mathrm{ABC}$ em comparação ao método por absorção. Acredita-se que este fato decorre da maior necessidade de entrada de dados.

Quanto ao TDABC observa-se que o método não é considerado de forma unânime uma evolução do método $\mathrm{ABC}$ tradicional. Para os docentes pesquisados, não há uma opinião uníssona se o método foi capaz de atingir alguns de seus objetivos, como a redução da complexidade e a diminuição da necessidade de tempo e recursos relacionados à sua implementação e manutenção. Do mesmo modo, observa-se que, na percepção dos respondentes, os quais foram alvos da pesquisa, o método apresenta limitações, não sendo capaz de explicar perfeitamente as atividades da empresa, além de não ser adequado a todos os tipos de organizações e ramos de atividade.

No que tange ao ABM, foi possível constatar que a literatura pesquisada apresenta poucos questionamentos ao seu respeito, recaindo o maior deles acerca da sua origem. Neste ponto, os docentes pesquisados convergem no entendimento de que o $\mathrm{ABM}$ pode ser considerado um sistema de gestão, do qual o ABC constitui uma extensão instrumental. $\mathrm{Ou}$ seja, o ABC é entendido como um instrumento de custeio. Opinião que corrobora estudos de Gosselin (1997) e Capusneanu e Martinescu (2010).

De forma geral, do total de questionamentos sobre os métodos baseados em atividades, apenas seis deles encontram-se consolidados por parte dos docentes e pesquisadores especialistas em custos. Este fato demonstra o quão controverso é o tema e a necessidade de uma maior gama de estudos na área. Por outro lado, é possível constatar haver um aparente trade-off entre complexidade e poder informacional: enquanto busca-se a redução da complexidade de implantação e a manutenção pela simplificação dos métodos (TDABC, por exemplo), isto tende a impactar na acurácia das informações fornecidas para a tomada de decisão.

Diante dos resultados encontrados neste estudo e com base na literatura pregressa notase que os questionamentos sobre os tópicos controversos dos métodos baseados em atividades estão parcialmente consolidados e que são necessárias novas investigações empíricas para observar a aderência dos mesmos nas organizações.

Por fim, deve-se interpretar os resultados com cautela. Mesmo que a academia seja um celeiro de conhecimento sobre teorias, conceitos e práticas de gestão, a opinião exclusiva de docentes e pesquisadores pode ter restringido a percepção ao campo teórico, uma vez que não há comprovação nesta pesquisa de que os mesmos tenham experiência prática com os métodos baseados em atividades. Ressalta-se ainda, que a análise do currículo lattes como critério para seleção da amostra pode ser considerada uma limitação em função da atualização frequente necessária, este fato pode ter levado a indagação de docentes e pesquisadores com especialidades distintas das exigidas por este estudo.

Sobre esta limitação, é importante salientar também que a área de custos é ampla. Mesmo que os critérios escolhidos para inclusão dos docentes na pesquisa tenham sido atendidos, o fato de um docente ter ministrado uma disciplina ou escrito um artigo relacionado a custos pode, em alguns casos, não significar que ele tenha um conhecimento profundo de um tema específico como o abordado neste estudo. Por fim, embora o número de docentes respondentes tenha sido considerado adequado de acordo com padrões recomendados para o tipo de instrumento utilizado, ele pode ter influenciado os testes utilizados, principalmente, no que tange à significância estatística destes.

Para futuros estudos sugere-se a realização de pesquisa similar com profissionais de mercado inseridos em empresas. Acredita-se que isto possibilitará novas percepções e possíveis comparações com os resultados obtidos neste estudo. A diferença entre teoria e prática pode lançar novos questionamentos e possibilidades interessantes para pesquisas subsequentes. No mais, cabe a sugestão de trabalhos com maior profundidade, capaz de responder as lacunas deixadas pelo estudo, inerentes ao método empregado. 


\section{REFERÊNCIAS}

BAIRD, K.; HARRISON, G.; REEVE, R. Adoption of activity management practices: a note on the extent of adoption and the influence of organizational and cultural factors. Management Accounting Research, v. 15, n. 4, p. 383-399, 2004.

BALAKRISHNAN, R.; LABRO, E.; SIVARAMAKRISHMAN, K. Product cost as decision aids: an analysis of alternative approaches (Part 1 e Part 2). Accounting Horizons, v. 26, n. 1, p. 1-41, 2012.

BOINA, T. M.; SOUZA, A. A.; AVELAR, E. A. Uma Análise bibliométrica de trabalhos acadêmicos sobre o Time-Driven Activity-Based Costing publicados em periódicos entre os anos de 2004 e 2013. Revista Universo Contábil, v.11, n. 4, p. 166-183, 2015.

BOKOR, Z.; SOMOGYI, R. Applying activity-based costing at logistics service providers. Periodica Polytechnica. Transportation Engineering, v. 43, n. 2, p. 98-105, 2015.

BYRNE, S. What determines ABC success in mature sites? Journal of Accounting \& Organizational Change, v. 7, n. 3, p. 259-277, 2011.

CAMPANALE, C.; CINQUINI, L.; TENUCCI, A. Time-driven activity-based costing to improve transparency and decision making in healthcare: a case study. Qualitative Research in Accounting \& Management, v. 11, n. 2, p. 165-186, 2014.

CAPUSNEANU, S; MARTINESCU, D. Convergence of ABC and ABM principles: guarantee of a performant management. Theoretical and Applied Economics, v. 10, n. 10, p. 93, 2010.

COELHO, P. S.; ESTEVES, S. P. The choice between a five-point and a ten-point scale in the framework of customer satisfaction measurement. International Journal of Market Research, v. 49, n. 3, p. 313-339, 2007.

COOPER, R.; KAPLAN, R. S. Measure costs right: make the right decisions. Harvard Business Review, v. 66, n. 5, p. 96-103, 1988.

COOPER, R.; KAPLAN, R.S. Activity-based systems: measuring the costs of resource usage. Accounting horizons, v. 6, n. 3, p. 1-13, 1992.

CRONBACH, L. J. Coefficient alpha and the internal structure of tests. Psychometrika, v. 16, n. 3, p. 297-334, 1951.

DALCI, I.; TANIS, V.; KOSAN, L. Customer profitability analysis with time-driven activitybased costing: a case study in a hotel. International Journal of Contemporary Hospitality Management, v. 22, n. 5, p. 609-637, 2010.

DIEHL, C. A.; GONÇALO, C. R; MARTINS, G. A. Estratégias competitivas em organizações de serviços no Brasil: uma análise descritiva. In: ENCONTRO NACIONAL DE ENGENHARIA DE PRODUÇÃO, 22 - ENEGEP. Curitiba, PR, de 23 a 25 de outubro de 2002. Anais. Curitiba, 2002.

EVERAERT, P.; BRUGGEMAN, W.; SARENS, G.; ANDERSON, S. R.; LEVANT, Y. Cost modeling in logistics using time-driven $\mathrm{ABC}$ : experiences from a wholesaler. International Journal of Physical Distribution \& Logistics Management, v. 38, n. 3, p. 172-191, 2008. 
FRIEDMAN, A. L.; LYNE, S. R. Activity-based techniques and the death of the beancounter. European Accounting Review, v. 6, n. 1, p. 19-44, 1997.

GHICAJANU, M. Activity based management-efficiency method of the management control systems. Annals of the University of Petroşani, Economics, v. 8, n. 1, p. 219-222, 2008.

GOSSELIN, M. The effect of strategy and organizational structure on the adoption and implementation of activity-based costing. Accounting, Organizations and Society, v. 22, n. 2, p. 105-122, 1997.

GUNASEKARAN, A; MCNEIL, R; SINGH, D. Activity-based management in a small company: a case study. Production, Planning \& Control, v. 11, n. 4, p. 391-399, 2000.

GUZMAN, L.; ABBELE, A. V.; VANDEWALLE, J.; VERHAAREN, H.; CATTRYSSE, D.Using Time-Driven Activity-Based Costing to support library management decisions: a case study for lending and returning processes. The Library, v. 84, n. 1, p. 1-23, 2014.

HAIR, J. F; BABIN, B.; MONEY, A.; SAMOUEL, P. Fundamentos de métodos de pesquisa em administração. Porto Alegre: Bookman, 2005.

HIXON, M. Activity-based management: its purpose and benefits. Management Accounting London, v. 73, n. 1, p. 30-30, 1995.

ISMAIL, N. A. Activity-based management system implementation in higher education institution: Benefits and challenges. Campus-Wide Information Systems, v. 27, n. 1, p. 40$52,2010$.

KACIUBA, G; SIEGEL, G. H. Activity-based management in a medical practice: a case study emphasizing the AICPA's core competencies. Issues in Accounting Education, v. 24, n. 4, p. 553-577, 2009.

KAPLAN, R. S.; ANDERSON, S. R. The innovation of time-driven activity-based costing. Journal of Cost Management, v. 11, n. 2, p. 5-15, 2007.

KAPLAN, R. S.; ANDERSON, S. R. Time-Driven Activity-Based Costing. Harvard Business Review, v. 01, p. 1-9, 2004.

KAPLAN, R. S.; NORTON, D. P. Mastering the management system. Harvard Business Review, v. 86, n. 1, p. 1-17, 2008.

KHOURY, C. Y.; ANCELEVICZ, J. Controvérsias acerca do sistema de custos ABC. Revista de Administração de Empresas, v. 40, n. 1, p. 56-62, 2000.

KIM, Y.W.; KIM, S.C. Cost analysis of information technology-assisted quality inspection using activity-based costing. Construction Management and Economics, v. 29, n. 2, p. 163172, 2011.

KONT, K; JANTSON, S. Activity-Based Costing (ABC) and Time-Driven Activity-Based Costing (TDABC): applicable methods for university libraries? Evidence Based Library and Information Practice, v. 6, n. 4, p. 107-119, 2011. 
KREN, L. Using Activity-Based management for cost control. Journal of Performance Management, v. 21, n. 2, p. 18-28, 2008.

LIN, W.C. Financial performance and customer service: an examination using activity-based costing of 38 international airlines. Journal of Air Transport Management, v 19, n. 1, p. 13$15,2012$.

MAIGA, A. S.; NILSSON, A.; JACOBS, F. A. Assessing the interaction effect of cost control systems and information technology integration on manufacturing plant financial performance. The British Accounting Review, v. 46, n. 1, p. 77-90, 2014.

MATELL, M. S.; JACOBY, J. Is there an optimal number of alternatives for Likert-scale items? Effects of testing time and scale properties. Journal of Applied Psychology, v. 56, n. 6, p. 506, 1972.

MILLER, J. G.; VOLLMANN, T. E. The hidden factory. Harvard business review, v. 63, n. 5, p. 142-150, 1985.

NULTY, D. D. The adequacy of response rates to online and paper surveys: what can be done? Assessment \& Evaluation in Higher Education, v. 33, n. 3, p. 301-314, 2008.

PHAN, T. N; BAIRD, K; BLAIR, B. The use and success of activity-based management practices at different organisational life cycle stages. International Journal of Production Research, v. 52, n. 3, p. 787-803, 2014.

RATNATUNGA, J.; MICHAEL, S. C.; BALACHANDRAN, K. R. Cost management in Sri Lanka: a case study on volume, activity and time as cost drivers. The International Journal of Accounting, v. 47, n. 3, p. 281-301, 2012.

ROCHA, W. Custeio baseado em atividades: mitos, falácias e possíveis verdades. In: Congresso Brasileiro de Custos, 1, São Leopoldo. Anais... São Leopoldo: ABC, 1994.

ROZTOCKI, N. Activity-based management for electronic commerce: a structured implementation procedure. Journal of theoretical and applied electronic commerce research, v. 5, n. 1, p. 1-10, 2010.

SANTANA, A; AFONSO, P. Analysis of Studies on Time-Driven Activity Based Costing (TDABC). International Journal of Management Science \& Technology Information, v. 1, n. 15, p. 133-157, 2015.

SCHOUTE, M. The relationship between product diversity, usage of advanced manufacturing technologies and activity-based costing adoption. The British Accounting Review, v. 43, n. 2, p. 120-134, 2011.

SCHULZE, M.; SEURING, S.; EWERING, C. Applying activity-based costing in a supply chain environment. International Journal of Production Economics, v. 135, n. 2, p. 716-725, 2012.

SHANK, J. K.; GOVINDARAJAN, V. A revolução dos custos. 2 ed. Rio de Janeiro: Campus, 1997. 
SOUZA, A. A.; AVELAR, E. A.; BOINA, T. M.; RAIMUNDINI, S. L.Análise da aplicabilidade do Time-driven Activity-based Costing em empresas de produção por encomenda. Revista Universo Contábil, v. 6, n. 1, p. 67-84, 2010.

STOUTHUYSEN, K.; SWIGGERS, M.; REHEUL, A. M.; ROODHOOFT, F. Time-driven activity-based costing for a library acquisition process: a case study in a belgian university. Library Collections, Acquisitions, and Technical Services, v. 34, n. 2-3, p. 83-91, 2010.

TARDIVO, G.; MONTEZEMOLO, D.; CORDERO, G. Using activity-based management to achieve excellence. Journal of Financial Management \& Analysis, v. 22, n. 1, p. 67-84, 2009.

TSAI, W. H, SHEN, Y. S.; LEE, P. L.; CHEN, H. C.; KUO, L.; HUANG, C. C.Integrating information about the cost of carbon through activity-based costing. Journal of Cleaner Production, v. 36, n. 1, p. 102-111, 2012.

VARILA, M; SEPPÄNEN, M; SUOMALA, P. Detailed cost modelling: a case study in warehouse logistics. International Journal of Physical Distribution \& Logistics Management, v. 37, n. 3, p. 184-200, 2007. 